\title{
CANAL NO YOUTUBE EM BUSCA DA PROMOÇÃO DA EDUCAÇÃO DE QUALIDADE NO BRASIL
}

Felipe Steigleder Gomes -f.steigleder.g@usp.br

Escola de Engenharia de Lorena - USP

Estrada Municipal do Campinho, s/n - Ponte Nova

12602-810 - Lorena - São Paulo

Maria Luiza Tarossi Martins Rosa-tarossimalu@usp.br

Escola de Engenharia de Lorena - USP

Estrada Municipal do Campinho, s/n - Ponte Nova

12602-810 - Lorena - São Paulo

Michael Douglas Angelo Rodrigues-michael_douglasangelo@usp.br

Escola de Engenharia de Lorena - USP

Estrada Municipal do Campinho, s/n - Ponte Nova

12602-810 - Lorena - São Paulo

Thaís Aparecida Galdino da Silva - thais_galdino@usp.br

Escola de Engenharia de Lorena - USP

Estrada Municipal do Campinho, s/n - Ponte Nova

12602-810 - Lorena - São Paulo

Resumo: A educação brasileira encontra-se num cenário crítico atualmente, com um ensino muito distante do ideal e escasso de oportunidades para grande parte da população. À vista disso, milhões de habitantes permanecem sem acesso à educação de qualidade e as desigualdades são perpetuadas no país. Assim, o objetivo deste projeto é a criação de um canal no YouTube com vídeos que abordem temas acadêmicos para analisar a exposição do conhecimento aliado ao entretenimento e promover o acesso à educação. Para isso, os alunos do primeiro ano de Engenharia de Produção da Escola de Engenharia de Lorena (USP), através de metodologia de natureza qualitativa e de finalidade exploratória, realizaram a construção de um canal, no qual foram publicados conteúdos e feitas análises a partir destes. Desse modo, os resultados foram satisfatórios, uma vez que foi criado um canal no YouTube respeitando os objetivos iniciais: conteúdo educacional de qualidade e entretenimento para os estudantes.

Palavras-chave: Educação de qualidade. YouTube. Entretenimento. Interdisciplinaridade. 


\section{INTRODUÇÃO}

A Organização das Nações Unidas (ONU), através da Agenda 2030, estipulou 17 objetivos de desenvolvimento sustentável, os quais servem de orientação para os países combaterem os desafios políticos, ambientais e econômicos. O objetivo 4, ponto de partida para realização dessa pesquisa, refere-se à Educação de Qualidade, buscando "assegurar a educação inclusiva e equitativa e de qualidade, e promover oportunidades de aprendizagem ao longo da vida para todos" (ONU, 2020a).

Os problemas na educação brasileira são notórios, à vista disso, o objetivo 4 da ONU está completamente de acordo com nossas necessidades de melhoria. O ensino está muito distante do ideal, uma vez que muitos não recebem oportunidades de aprendizagem decentes ao longo da vida e não têm acesso ao conhecimento. Dessa forma, as desigualdades são perpetuadas no país, e a educação de qualidade apresenta-se como uma concepção quase utópica para a maior parte da população. Como agravante, na maioria das escolas brasileiras, há o predomínio de um ensino padronizado, no qual o professor repassa o conteúdo, e o aluno apenas absorve, de forma monótona, através da cópia da matéria transmitida pelo docente. Assim, tal padronização resulta em falta de interesse e desmotivação dos alunos para com o conhecimento, fazendo com que, na maioria das vezes, não aprendam a matéria lecionada.

Diante de um sistema educacional ineficaz e pouco inclusivo, são necessárias iniciativas que incentivem a disseminação de conhecimento de qualidade para todos, tornando pertinente a criação de um canal no YouTube com vídeos educativos, interdisciplinares e dinâmicos.

Tendo em vista a teoria de Glasser, a qual apresenta uma pirâmide de aprendizagem com diferentes percentuais de absorção de conhecimento conforme distintos métodos de ensino, nota-se que o envolvimento de modos e sentidos diversos pode funcionar como um facilitador para a aprendizagem (GLASSER, 2001). A partir disso, através da criação de um canal (Canal Educare), busca-se garantir ao aluno acesso a um conteúdo de qualidade e atraente para entretêlo enquanto aprende. Para isso, objetiva-se produzir vídeos dinâmicos que tornem a transmissão do conhecimento mais efetiva, estimulando o gosto pelo aprendizado.

Este projeto foi desenvolvido por quatro estudantes do primeiro ano do curso de Engenharia de Produção da Escola de Engenharia de Lorena da Universidade de São Paulo na disciplina de Projeto Integrado de Engenharia de Produção I, na qual foi designado como tema "Educação de Qualidade". Visando o desenvolvimento de competências técnicas (a exemplo da gestão de projetos) e transversais (como trabalho em equipe, comunicação e relacionamento interpessoal), a disciplina segue a metodologia de "project based learning", isto é, aprendizagem baseada em projetos, na qual os alunos elaboram projetos procurando resolver problemas reais. Por conta da pandemia do Covid-19, a disciplina foi ministrada à distância, através de videoconferências, durante o primeiro semestre de 2020 (JOLLANDS et al, 2012; LEHMANN et al, 2008; PEREIRA et al, 2018).

\section{REVISÃO DE LITERATURA}

\subsection{Aplicação do audiovisual na educação}

O ensino tradicional é o mesmo há séculos, porém, métodos inovadores podem revolucionar a educação. Diante disso, a tecnologia é uma forma de aprimorar a qualidade da educação, pois proporciona novos caminhos para o ensino e aprendizagem, além de novas metodologias. Dessa forma, essa ferramenta, cada vez mais presente na educação, poderá despertar a curiosidade e promover novas descobertas, estimulando novas experiências, e, 
assim, construindo competências e contribuindo para o desenvolvimento de muitos estudantes (ANTUNES, 2015).

Nesse sentido, o uso do audiovisual é uma estratégia que pode revolucionar o ensino atual das escolas brasileiras. No entanto, esse recurso não irá excluir ou minimizar a função do professor na sala de aula, pelo contrário, pode servir como forte aliado. Além de ser estimulante para os alunos, ele pluraliza o processo educacional, apresentando outra possibilidade de diálogo e trazendo diferentes olhares. Logo, com dinâmica do mundo moderno e da juventude, serve de estímulo não só ao aluno, mas também ao professor (BONETTI, 2008; FERREIRA, 2010). Eurico Costa Ferreira (2010), no trecho a seguir, completa esse ponto de vista: "o uso de novas tecnologias pode ser um recurso pedagógico fundamental, e dessa forma, além do livro didático, outros recursos didáticos passam a ser utilizados pelos professores no processo de ensino-aprendizagem".

A sociedade em que vivemos está repleta de imagens e sons, entre outros estímulos. É um mundo de comunicação, em que os sentidos, as emoções e a razão são ativados, interagindo e criando a nossa cultura e humanidade. Como a tecnologia está cada vez mais inserida na sala de aula e nos conceitos pedagógicos de escolas e professores, uma opção viável e eficiente é a utilização de vídeos para a potencialização do aprendizado dos alunos.

O vídeo além de mudar os cenários das aulas, pode desenvolver no aluno atitudes críticas (sic) em relação a aquilo que lhe é apresentado. Com o vídeo existem possibilidades de aumentar suas percepções através das linguagens, e desta forma poderá despertar a construção de ideias, de argumentos que podem levar a interação e a aproximação de todos. (SANTOS, 2015, p. 23)

Em experimento com alunos do $1^{\circ}$ ano do Ensino Médio de São Paulo, Marcelo de Carvalho Bonetti (2008) apresentou conteúdos acadêmicos de duas formas: um vídeo semelhante a uma aula tradicional e outro com um formato televisivo. O resultado mostrou que os dois contribuem para o aprendizado de conceitos científicos e que, quando aliados, promovem melhoras significativas no aprendizado desses conceitos, formando uma maneira de aprendizagem distinta e complementar. Dessarte, nota-se que o conteúdo acadêmico deve ser apresentado com o uso dos dois métodos (um mais formal e outro mais divertido) para melhor retenção do conhecimento.

Dessa forma, encontramos indícios de que os vídeos prazerosos devem ser mais valorizados no ensino, pois são tão educativos quanto vídeos monótonos. Também identificamos que realizadores de audiovisuais educativos devem ter consciência da cooperação que ocorre quando se mesclam momentos de sistematização e momentos de prazer num audiovisual, o que pode ser contemplado por professores que utilizem vídeos de naturezas distintas. (BONETTI, 2008, p. 3)

\subsection{Uso da internet no ambiente acadêmico}

No mundo globalizado, a internet tornou-se a maior ferramenta para compartilhar informações, além de rápida, é também muito acessível. De acordo com um estudo do IBGE (Instituto Brasileiro de Geografia e Estatística), em 2017, 74,9 \% dos domicílios brasileiros tinham acesso a essa rede, mostrando seu alto alcance. A inserção desse recurso no ambiente acadêmico pode proporcionar melhor desenvolvimento na aprendizagem dos alunos, uma vez que ela disponibiliza grande quantidade de artigos, vídeos, e textos acadêmicos. Se utilizados de maneira correta, em prol do conhecimento, esses recursos podem facilitar bastante o processo de ensino e aprendizagem, como defendido no trecho:

Uma educação tradicional centrada em uma metodologia passiva que já não atrai os estudantes, gerando desinteresse, evasão e indisciplina, precisa ser 
repensada e alterada. Os jovens estudantes que ocupam as carteiras escolares nesse último período da educação básica, são estudantes que possuem amplo acesso a tecnologias digitais e é totalmente natural e desejável para eles que a escola envolva processos tecnológicos no ensino e na aprendizagem. (GODOI, 2020, p. 19)

Cada pessoa possui a sua forma de aprender, e não existe um modelo ideal que irá englobar todas elas, contudo, vários estudos já apontam técnicas mais efetivas de aprendizado.

\subsection{Teoria de Glasser}

O psiquiatra estadunidense William Glasser elaborou uma teoria na qual defende as diferentes formas de aprendizado e sua porcentagem de efetividade (GLASSER, 2001). Segundo ele, a retenção do conhecimento se dá, basicamente, da seguinte forma:

- $10 \%$ por meio da leitura;

- $20 \%$ por meio da escrita;

- $50 \%$ por meio da observação e escuta;

- $70 \%$ por meio da discussão com outros colegas;

- $80 \%$ por meio da prática;

- $95 \%$ por meio do ensino.

Através de uma análise das formas de aprendizado na teoria de Glasser, é possível observar que os métodos mais efetivos fazem parte de metodologias ativas de aprendizagem. Ao envolver diferentes modos e sentidos, o aluno não recebe somente conteúdo de forma passiva e assimila de forma mais eficaz o conteúdo. Portanto, nota-se a necessidade de testar novas formas de ensino, envolvendo distintas habilidades, para explorar o máximo possível dos estudantes brasileiros.

\section{METODOLOGIA}

A aprendizagem baseada em projetos foi a metodologia empregada na disciplina PIEP 1, a qual visava o aprimoramento de competências transversais e o desenvolvimento da competência técnica de gestão de projetos. Através de atividades como discussões entre o grupo, elaboração de relatórios e produção de vídeos, a habilidade de comunicação foi bastante explorada, assim como o trabalho em equipe.

O projeto seguiu uma metodologia de natureza qualitativa, na qual dados são usados para coletar informações que buscam não só medir um tema, mas também descrevê-lo, com base em opiniões e pontos de vista. Nesse caso, parte-se de um marco teórico e uma metodologia preestabelecidos para, depois, determinar os instrumentos de coleta de dados e analisar os resultados obtidos. Assim, envolvendo a observação desses resultados e sua discussão, essa abordagem proporciona uma compreensão mais detalhada das perguntas da pesquisa (GERHARDT; SILVEIRA, 2009; GUERRA, 2014).

Ademais, do ponto de vista conceitual, trata-se de um projeto básico, tendo assim objetivo exploratório. Sua abordagem deu-se principalmente através do levantamento de informações para melhor conhecimento do assunto, de maneira a proporcionar maior familiaridade dos participantes em relação ao mesmo, tornando-o mais explícito. Por conta do objetivo de uma visualização geral do problema, a pesquisa exploratória é flexível quanto ao planejamento, permitindo a análise de diferentes aspectos do tema (GERHARDT; SILVEIRA, 2009).

Em acordo com a metodologia de natureza qualitativa e objetivo exploratório, no projeto desenvolvido, a coleta de dados foi realizada principalmente através da observação de comentários e visualizações nos vídeos postados e nas redes sociais. Diante disso, eram 
realizadas a análise das possíveis melhorias e a discussão de novas ideias para o canal do YouTube. Durante o desenvolvimento do projeto, os integrantes, naturalmente, tiveram que se aprofundar no estudo de métodos educacionais e de outros aspectos relacionados à educação de qualidade para aprimorar o conteúdo disponibilizado no YouTube.

\section{DESENVOLVIMENTO DO PROJETO}

Por conta do contexto de isolamento social (devido à pandemia do covid-19), o planejamento e a execução do projeto foram realizados por meio da internet, com videoconferências e discussões no grupo do WhatsApp. Visto que o projeto já seria executado online, utilizando redes sociais e o YouTube, o isolamento social não foi muito prejudicial, afetando apenas a divulgação do canal, a qual poderia também ser realizada em escolas.

O projeto desenvolveu-se conforme um processo de criação e postagem de conteúdo seguido de análise de feedback. Primordialmente, foi realizada uma pesquisa bibliográfica com artigos relacionados à educação de qualidade, tecnologia e entretenimento. Dessa forma, estabelecida uma base teórica, iniciou-se a parte prática do projeto, isto é, a produção de vídeos. A partir disso, a execução do projeto divide-se em três etapas: elaboração dos vídeos, divulgação e análise de feedback.

\subsection{Produção de um vídeo}

Primeiramente, é realizada uma pesquisa sobre fatos, eventos históricos ou outros tópicos que instigam a curiosidade dos alunos. A partir disso, o grupo delimita um tema central, o qual norteará a elaboração do roteiro. Em seguida, um narrador é selecionado para a gravação do áudio explicativo. Depois, a equipe separa uma coletânea de imagens, que serão posteriormente inseridas no vídeo, tornando-o mais atrativo e didático. $\mathrm{Na}$ edição, utilizando o aplicativo KineMaster, elabora-se a animação, relacionando as imagens e a narração. Por fim, é adicionada ao vídeo uma curta participação de um professor convidado, de forma a acrescentar conteúdo e credibilidade.

\subsection{Divulgação}

O processo de divulgação é realizado através das redes sociais. A princípio, o grupo criou contas nas redes sociais (Facebook e Instagram) para o canal, denominado Educare. Assim, com o apoio de amigos, é realizada a divulgação do canal e seus vídeos através de postagens nessas plataformas.

\subsection{Análise de feedback}

A coleta de dados a respeito do feedback do público, diante dos vídeos postados, é feita mediante análise de comentários nos vídeos e nas redes sociais. Além disso, a ferramenta "Analytics" do YouTube permite uma visão mais aprofundada da interação das pessoas com os vídeos, exibindo dados como a idade do público, visualizações ao longo do tempo e a origem dos acessos.

\section{RESULTADOS E DISCUSSÃO}

No início do projeto, esperava-se que os estudantes se interessassem pelo canal e pelos vídeos, compreendendo os conhecimentos abordados e se entretendo com os mesmos. Desse modo, pretendia-se mostrar que o conhecimento, se abordado com uma linguagem mais 


\section{COBENGE (C) COBENGE 2020 da ABENGE}

próxima do aluno e de forma mais dinâmica, poderia proporcionar mais interesse e motivação para os estudantes, facilitando o aprendizado. Finalmente, objetivava-se mostrar que a exploração da linguagem audiovisual de diferentes maneiras enriquece a busca pelo conhecimento.

Após a conclusão do projeto, os resultados obtidos foram favoráveis, já que foi possível criar um canal no YouTube respeitando os anseios iniciais: conteúdo educacional de qualidade e entretenimento para os estudantes. A respeito do canal em si, um número satisfatório de inscritos foi alcançado, como ilustra a Figura 1, além de consideráveis quantidades de visualizações e curtidas no primeiro vídeo, como pode ser observado na Figura 2. Outrossim, os comentários foram bastante positivos, com vários elogios e incentivos, como mostra a Figura 3.

Figura 1 - Canal no YouTube

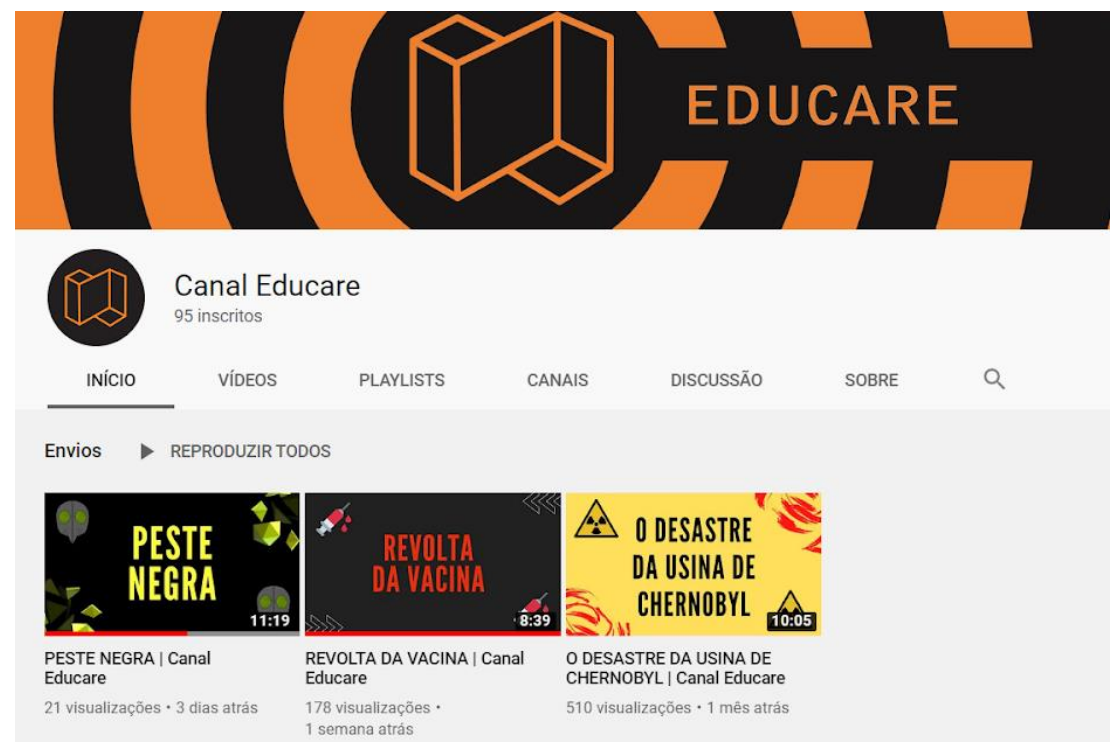

Fonte: Autores

Figura 2 - Gráfico de visualizações em função do tempo

Video origem do tráfego - Mais -

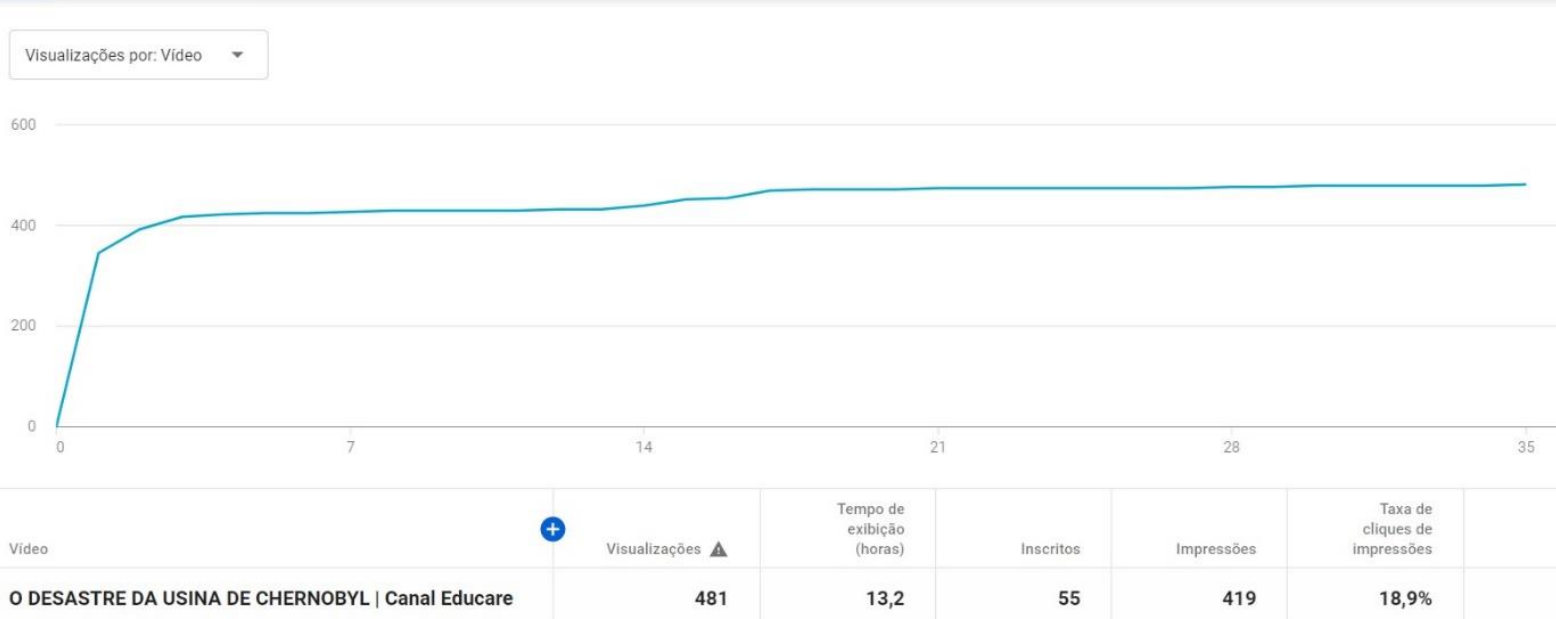

Fonte: Autores 
Figura 3 - Comentários do primeiro vídeo
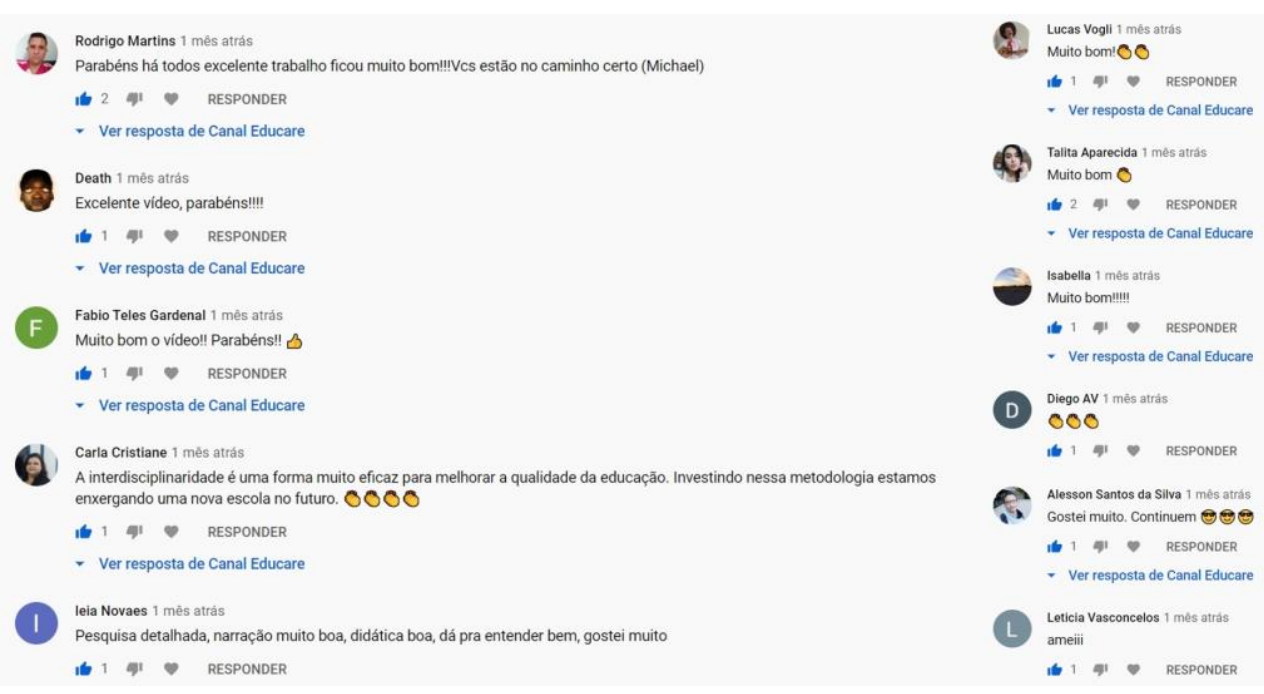

Fonte: Autores

Quanto à divulgação, inicialmente, esperava-se alcançar um grande número de alunos, através da ajuda de docentes, que ajudariam ao expor os vídeos na sala aula de aula. Dessa maneira, os alunos teriam ainda mais contato com o canal. Entretanto, devido à pandemia do covid-19, as aulas presenciais foram suspensas, e a divulgação ocorreu apenas por via das redes sociais (Instagram e Facebook, respectivamente ilustrados pelas Figuras 4 e 5).

Figura 4 - Página no Instagram
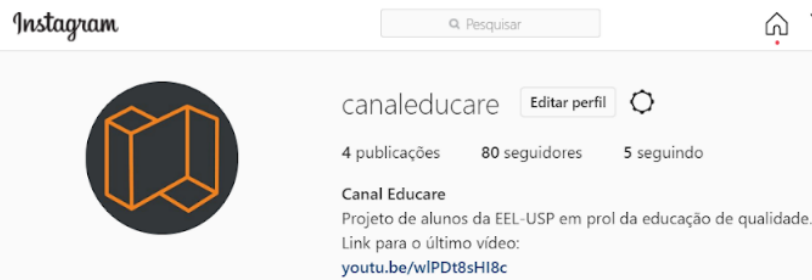

Fonte: Autores

Figura 5 - Página no Facebook

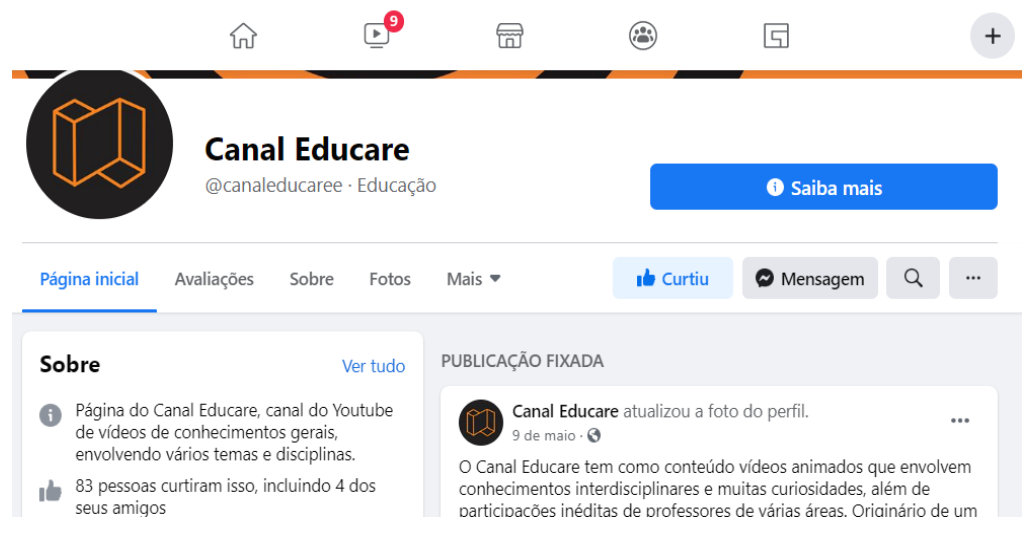

Fonte: Autores 
A partir do uso do recurso Analytics do Youtube, foi possível visualizar informações mais específicas dos vídeos, como os tipos de origem de tráfego e as visualizações diárias dos vídeos. As Figuras 6 e 7, extraídas dessa ferramenta, ilustram a origem do tráfego do primeiro vídeo, mostrando que a maior parte das visualizações têm origem externa ao YouTube. Fica claro, portanto, que as redes sociais do canal tiveram grande eficácia na divulgação.

Figura 6 - Gráfico dos tipos de origem de tráfego

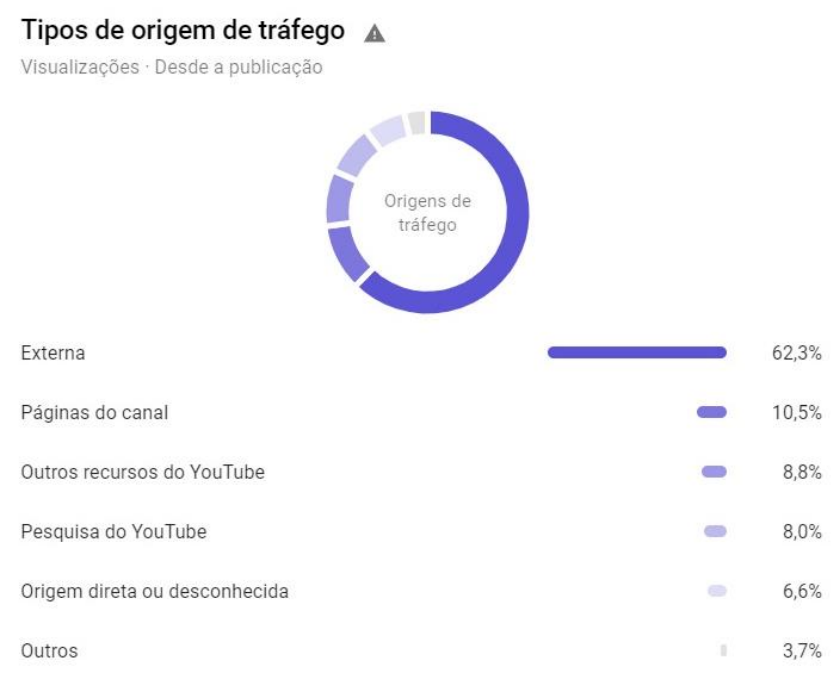

Fonte: Autores

Figura 7 - Influência das redes sociais no tráfego externo

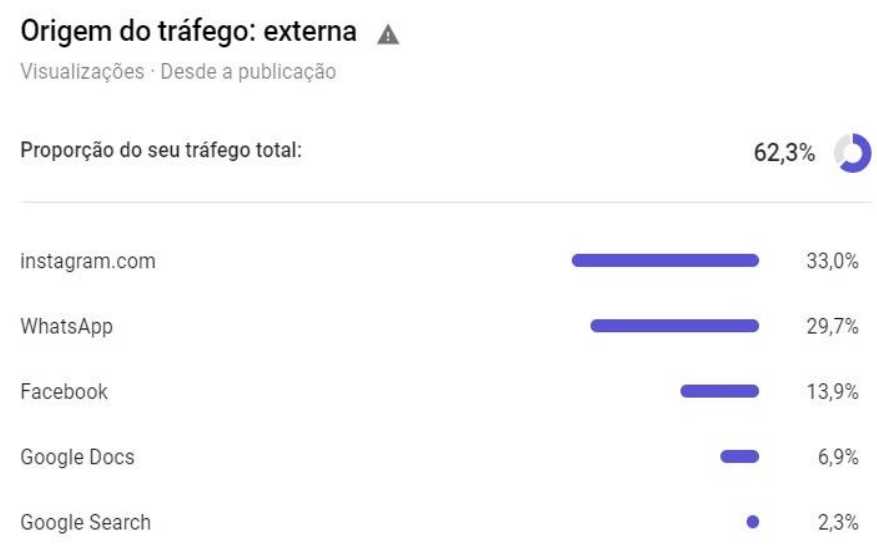

Fonte: Autores

\section{CONCLUSÃO}

O projeto teve como objetivo encontrar maneiras de auxiliar na promoção da educação de qualidade no Brasil. Considerando que o canal no YouTube se apresenta como mais um recurso para estudantes de todo o país acessarem conhecimento, pode-se afirmar que a meta foi atingida. Ademais, vale ressaltar o emprego da interdisciplinaridade no projeto, como uma ferramenta de ensino para assimilação de conteúdos de diferentes áreas e relacionamento de informações normalmente exploradas isoladamente nas escolas. 
Dessa forma, foram realizadas diversas contribuições para a educação brasileira, servindo de exemplo para futuras iniciativas e pesquisas que busquem explorar o âmbito educacional, objetivando a educação de qualidade no país. Dentre essas contribuições, convém destacar o incentivo ao interesse e à motivação dos estudantes, uma vez que o canal apresenta uma maneira dinâmica e lúdica de ensino. Outrossim, diante da disponibilização gratuita de conteúdo educativo de qualidade para todos no YouTube, professores podem utilizar os vídeos como recurso didático em sala de aula, empregando-os para introduzir, fixar e sintetizar temas.

Quanto à execução do projeto, foi adotada uma metodologia de natureza qualitativa e objetivo exploratório, que permitiu uma melhor compreensão do assunto por parte dos integrantes do grupo. Durante a elaboração da pesquisa, foram desenvolvidas habilidades de gestão de grupos e competências transversais - principalmente trabalho em equipe -, as quais têm relevância na formação dos autores.

\section{Agradecimentos}

Os autores agradecem aos professores: Álvaro Henrique Mello José, Gustavo Baviera e Taciana Ferreira de Andrade pela participação e contribuição nos vídeos. Um agradecimento especial para o professor Marco Antônio Carvalho Pereira, que nos orientou na conduta da disciplina, e à tutora Ana Luiza do Nascimento, que nos auxiliou em todas as etapas do projeto.

\section{REFERÊNCIAS}

ANTUNES, Kate Francisca da Silva. Os benefícios do uso pedagógico dos recursos audiovisuais em sala de aula, segundo estudantes do centro de ensino médio 804 do recanto das EMAS. Monografia (Curso de Especialização em Coordenação Pedagógica) Universidade de Brasília, Brasília, 2015.

BONETTI, Marcelo de Carvalho. A linguagem de vídeos e a natureza da aprendizagem. 172 f. Dissertação (Mestrado) - Universidade de São Paulo. Instituto de Física - Departamento de Física Experimental, São Paulo, 2008.

FERREIRA, Eurico Costa. O uso dos audiovisuais como recurso didáctico. 75 f. Dissertação (Mestrado) - Universidade do Porto. Faculdade de Letras, Porto, 2010.

GERHARD, Tatiana Engel; SILVEIRA, Denise Tolfo. Métodos de pesquisa. $1^{\text {a }}$ edição, Porto Alegre: Editora da Ufrgs, 2009.

GLASSER, W. Teoria da Escolha: uma nova psicologia de liberdade pessoal. $1^{\text {a }}$ edição, São Paulo: Mercuryo, 2001.

GODOI, Francis Pegorer. Ensino ativo de filosofia: aliando a pedagogia do conceito com tecnologias digitais. 2020. 90 f. Dissertação (Mestrado) - Curso de e Pós-graduação em Docência Para A Educação Básica, Universidade Estadual Paulista, Bauru, 2020.

GUERRA, Elaine Linhares de Assis. Manual de pesquisa qualitativa. $1^{\text {a }}$ edição, Belo Horizonte: Editora Grupo Anima Educação, 2014.

INSTITUTO BRASILEIRO DE GEOGRAFIA E ESTATÍSTICA (IBGE). Disponível em: <https://biblioteca.ibge.gov.br/visualizacao/livros/liv101631_informativo.pdf> Acesso em: 8 de abril de 2020. 
JOLLANDS, M., JOLLY, L.; MOLYNEAUX, T. Project-based learning as a contributing factor to graduates' work readiness. European Journal of Engineering Education. 22 mar. 2012, p. 143-154.

LEHMANN, M.; CHRISTENSEN, P.; DU, M. \& THRANE, M. Problem-oriented and projectbased learning (POPBL) as an innovative learning strategy for sustainable development in engineering education. European Journal of Engineering Education. 25 jun. 2008, p. 283295.

Organização das Nações Unidas. Agenda 2030. ONU. Disponível em: <https://nacoesunidas.org/pos2015/ods11/> Acesso em: 26 abr. 2020.

PEREIRA, M. A. C.; LENCO, J; REY, M. Modelo de competências de uma disciplina de projeto de primeiro ano de um curso de engenharia de produção. 2018. Disponível em: <http://www.abenge.org.br/sis_artigos.php> Acesso em: 28 abril 2020.

SANTOS, Rosiane de Jesus. Uma Taxionomia para o uso de Vídeos Didáticos para o Ensino de Matemática. 2015. 132 f. Dissertação (Mestrado) - Curso de Educação Matemática, Universidade Federal de Juiz de Fora. Juiz de Fora. 2015.

\title{
YOUTUBE CHANNEL TO FORWARD QUALITY EDUCATION IN BRAZIL
}

\begin{abstract}
The Brazilian education is currently in a critical scenario, far from ideal and scare of opportunities for a great part of population. Therefore, millions of inhabitants remain without access to quality education and the inequalities are perpetued in the country. Thus, the objective of this project is the creation of a YouTube channel with videos related to academic topics to analyze the exposure of knowledge combined with entertainment and promote access to education. To this end, the students of the first year of production engineering at the Escola de Engenharia de Lorena (USP), through the metodology of qualitative nature and exploratory purpose, developed a YouTube channel, named Canal Educare, in which contents were published and analyzed. Therefore, the results were sactisfactory, since the YouTube channel was created respecting the initial objectives: quality educative content and entertainment for students.
\end{abstract}

Keywords: Quality education. YouTube. Entertainment. Interdisciplinarity. 